\title{
Thoracoscopic lobectomy for pulmonary vein occlusion after radiofrequency catheter ablation of atrial fibrillation
}

\author{
Chien-Ming Lo, Hung-l Lu, Yen-Yu Chen and Jen-Ping Chang*
}

\begin{abstract}
Background: Radiofrequency catheter ablation for paroxysmal atrial fibrillation is well established but not drawback free. Pulmonary vein stenosis is one of the complications and usually treated with stenting with the disadvantages of high re-stenosis rate and anticoagulant dependence.

Case presentation: Herein, we present the case of a 47 year-old lady, who suffered from fever and hemoptysis due to right inferior pulmonary vein occlusion after radiofrequency catheter ablation for paroxysmal atrial fibrillation.

Eventually, thoracoscopic right lower lung lobectomy was inevitable with satisfactory result.

Conclusions: Pulmonary vein stenosis is a major complication after radiofrequency ablation of atrial fibrillation. High suspicion and early detection in patients with pulmonary manifestations are mandatory for salvage the injured lung in early. If delayed, surgical resection of the involved lung, especially through the thoracoscopic approach will eradicate the problem with minimal complication.
\end{abstract}

Keywords: Atrial fibrillation, Radiofrequency catheter ablation, Thoracoscopic lobectomy

\section{Background}

Radiofrequency catheter ablation is one of the recommended therapeutic options for the paroxysmal atrial fibrillation with low complication rate and promising success rate. However, pulmonary vein stenosis is one of the major complications of this procedure and could be fatal [1]. Hemoptysis, dyspnea, intractable cough, and recurrent pneumonia are the most common manifestations [2]. Radical procedure like lobectomy may be necessary in the case of medical treatment failure, recurrent pneumonia or sepsis $[3,4]$. We reported on a 47 year-old lady with recurrent pulmonary events after radiofrequency catheter ablation of paroxysmal atrial fibrillation. The diagnosis of right inferior pulmonary vein occlusion was delayed and eventually, thoracoscopic right lower lung lobectomy was inevitable with satisfactory result.

\footnotetext{
* Correspondence: c9112772@adm.cgmh.org.tw

Department of Thoracic and Cardiovascular Surgery, Kaohsiung Chang Gung Memorial Hospital, Chang Gung University, College of Medicine, 123 Tapei Rd, Niaosung District, Kaohsiung City 833, Taiwan, Republic of China
}

\section{Case presentation}

A 47 year-old barber lady with three-year history of paroxysmal atrial fibrillation was admitted on 2 June 2013 for recent incremental attacks of palpitation. Physical examination and laboratory tests were all unremarkable. On 4 June 2013, the electrophysiological study using EnSite $^{\mathrm{TM}}$ Velocity $^{\mathrm{TM}}$ (St. Jude Medical One St. Jude Medical Drive, St. Paul, MN 55117 USA) through the transseptal approach confirmed that the paroxysmal atrial fibrillation was originating from the right superior and inferior pulmonary veins. Subsequently, 20-30 Watts $\left(45^{\circ}\right.$ Celsius for 30 to $\left.40 \mathrm{~s}\right)$ of radiofrequency energy was applied to atrial locations of the four pulmonary veins utilizing a $7 \mathrm{~F}$ quadripolar irrigating ablation catheter. The successful ablation was confirmed by complete antegrade and retrograde electrophysiological study using incremental pacing and extrastimulus technique during isoproterenol administration. The patient was discharged six days later with uneventful course until 10 September 2013, a febrile episode associated with productive cough and mild dyspnea happened. Right lower lobe pneumonia was found by chest roentgenography and computed tomography. The patient was 
discharged in improved condition after antibiotic treatment for two weeks.

Hemoptysis and chest discomfort occurred 23 November 2013. An emergent chest roentgenogram revealed right lower lung consolidation. The bronchoscopic study was unremarkable. A cardiac computed tomographic angiogram was performed on the presumption of iatrogenic pulmonary vein injury and revealed right inferior pulmonary vein occlusion on 28 November 2013 (Fig. 1a and b). Because of the totally occluded pulmonary vein, stenting was aborted due to high technical demand, high restenosis rate, and need of almost life-long anticoagulant therapy. On 10 December 2013, six months since the radiofrequency ablation of the paroxysmal atrial fibrillation, thoracoscopic right lower lobectomy was performed with four-port technique. Severe inflammatory adhesion of the right pulmonary hilar region and totally fibrotic right inferior pulmonary vein were noted. The postoperative course was uneventful and the patient was discharged on eleventh postoperative day. Chest roentgenogram was followed every three months and cardiac computed tomographic angiogram was followed every six months during the follow-up period. The patient has been free from both pulmonary and cardiac events 18 months postoperatively and 24 months after the radiofrequency ablation of the atrial fibrillation.

On the microscopic examination, extensive hemorrhagic necrosis with acute and chronic inflammatory cell infiltration, interstitial fibrosis, and vascular thrombosis were noted around the right inferior pulmonary vein.

\section{Discussion}

Besides the medical rate control and surgical ablation, radiofrequency catheter ablation is another therapeutic option for the paroxysmal atrial fibrillation with intractable symptoms. The procedure related overall complication rate is $6.29 \%$. Among them, the cardiac complications include myocardial infarction, pericardial complications and various iatrogenic cardiac injuries had been reported. The pulmonary vein injury is one of the major adverse events of this procedure [1-3].

Pulmonary vein stenosis can be under-recognized because the manifestations are often obscure in the early stage or in cases with limited involvement. Repetitive or progressive deterioration of non-specific respiratory manifestations suggest that the iatrogenic pulmonary vein stenosis getting significant. The first 2 cases of pulmonary vein stenosis after radiofrequency ablation of atrial fibrillation were successfully treated with balloon dilation [5]. Since then, this complication is usually treated with percutaneous transluminal pulmonary vein balloon dilatation or stenting with the demerits of significant re-stenosis rate and need of long-term antithrombotic therapy [2]. In the limited cases with irreversible occlusion with significant symptoms, dilation and stenting can be not effective and eventually requiring pulmonary resection $[4,6,7]$.

In our patient, because of the totally occluded pulmonary vein, the percutaneous transluminal approach was aborted and the surgical resection of the injured lung would be the final option. Certainly, this method would eradicate the lesion on the cost of sacrifice of pulmonary capacity. Otherwise, although technically demanding, surgical pulmonary venous angioplasty might be another salvage option. However, in the chronic case with severe inflammatory adhesion, this procedure might be impossible. Furthermore, the injury created by the radiofrequency energy was reported to result in irreversible venous and arterial medial thickening and intimal hyperplasia throughout the lung with consolidation that prohibited the lung sparing strategies [6].

\section{Conclusion}

In conclusion, pulmonary vein stenosis is a major complication after radiofrequency ablation of atrial fibrillation. High suspicion and early detection in patients with pulmonary manifestations are mandatory for salvage the injured lung in early. If delayed, surgical resection of the

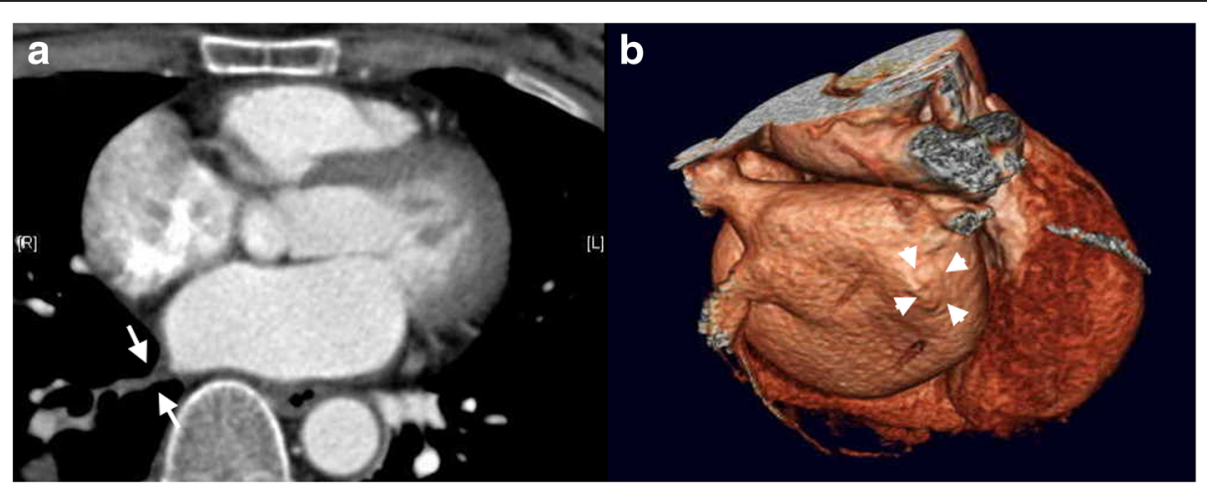

Fig. 1 a: A cardiac computed tomographic angiogram revealed right inferior pulmonary vein occlusion (between arrows). b: A three-dimensional cardiac computed tomographic angiogram showed absent right inferior pulmonary vein (arrow heads) 
involved lung, especially through the thoracoscopic approach will eradicate the problem with minimal complication.

\section{Informed consent}

Written informed consent was obtained from the patient for publication of this Case report and any accompanying images. A copy of the written consent is available for review by the Editor-in-Chief of this journal. This case study was approved by Institutional Review Board for Kaohsiung Chang Gung Memorial Hospital (104-7429B).

\section{Competing interests}

The authors declare that they have no competing interests.

\section{Authors' contributions}

All authors participated in the design of the case report and coordination and helped to draft the manuscript. All authors read and approved the final manuscript.

\section{Disclosures}

The authors have no funding, financial relationships or conflicts of interest disclosure.

Received: 17 November 2015 Accepted: 10 January 2016

Published online: 19 January 2016

\section{References}

1. Gupta A, Perera T, Ganesan A, Sullivan T, Lau DH, Roberts-Thompson KC, et al. Complications of catheter ablation of atrial fibrillation: a systematic review. Circ Arrhythm Electrophysiol. 2013:6:1082-8.

2. Holmes Jr DR, Monahan KH, Packer D. Pulmonary vein stenosis complicating ablation for atrial fibrillation: clinical spectrum and interventional considerations. JACC Cardiovasc Interv. 2009;2:267-76.

3. Deshmukh A, Patel NJ, Pant S, Shah N, Chothani A, Mehta K, et al. In-hospital complications associated with catheter ablation of atrial fibrillation in the United States between 2000 and 2010: analysis of 93801 procedures. Circulation. 2013:128:2104-12

4. Libretti L, Ciriaco P, Zannini P. Pulmonary vein stenosis requiring lobectomy after radiofrequency catheter ablation for atrial fibrillation. J Cardiovasc Surg. 2012;53:821-3

5. Robbins IM, Colvin EV, Doyle TP, Kemp WE, Loyd JE, McMahon WS, et al. Pulmonary vein stenosis after catheter ablation of atrial fibrillation. Circulation. 1998:98:1769-75.

6. Yang HM, Lai CK, Patel J, Moore J, Chen PS, Shivkumar K, et al. Irreversible intrapulmonary vascular changes after pulmonary vein stenosis complicating catheter ablation for atrial fibrillation. Cardiovasc Pathol. 2007;16:51-5.

7. Steliga MA, Ghouri M, Massumi A, Reul RM. Lobectomy for pulmonary vein occlusion secondary to radiofrequency ablation. J Cardiovasc Electrophysiol. 2010;21:1055-8.

Submit your next manuscript to BioMed Central and we will help you at every step:

- We accept pre-submission inquiries

- Our selector tool helps you to find the most relevant journal

- We provide round the clock customer support

- Convenient online submission

- Thorough peer review

- Inclusion in PubMed and all major indexing services

- Maximum visibility for your research

Submit your manuscript at www.biomedcentral.com/submit 